\title{
Geomagnetic gradient-assisted evolutionary algorithm for long-range underwater navigation
}

\author{
Jiayu Zhang, Tao Zhang, Hyo-Sang Shin, Jian Wang, Chen Zhang
}

\begin{abstract}
Extensive research results have shown that animals like pigeons and turtles can use geomagnetic information for long-distance migration and homing. This article studies the bionic navigation method inspired by magnetotaxis behavior without prior knowledge. The problem of bionic geomagnetic navigation is generalized as an autonomous search of navigation path under the excitation of geomagnetic environment. The geomagnetic gradient-assisted evolutionary algorithm for long-range underwater navigation is proposed. In order to optimize the navigation path, the heading angle predicted by the geomagnetic gradient is used to constrain the sample space in the evolutionary algorithm. Then, according to the principle of multi-parameter simultaneous convergence, the evaluation function is improved to enhance the reliability and accuracy of the navigation path. Simulations of the algorithm before and after improvement are carried out based on the data retrieved from the Enhanced Magnetic Model (EMM). The performance of the improved method is evaluated and verified in the case of the area with normal geomagnetic field (GF), geomagnetic anomaly area and multiple destinations. The simulation results show that the search efficiency and the straightness of the navigation path are greatly improved. The reason is that the constraint of sample space reduces the randomness in the process of navigation path search, and the improved evaluation function can evaluate the quality of samples more accurately. The improved algorithm also has good performance in the geomagnetic anomaly area, which indicates the potential application in the future.
\end{abstract}

Index Terms - Evolutionary algorithm, Bionic geomagnetic navigation, Multi-objective optimization, Navigation path searching, Geomagnetic field

This work was supported in part by National Natural Science Foundation of China 52071080, the Inertial Technology Key Lab Fund under Grant 614250607011709, in part by the Fundamental Research Funds for the Central Universities under Grant 2242020k1G009, Key Laboratory Fund for Underwater Information and Control under Grant 614221805051809 , and in part by the Jiangsu Key Laboratory Fund for Green Ship Technology under Grant 2019Z01, Remaining funds cultivation project of National Natural Science Foundation of Southeast University under Grant 9S20172204

J. Zhang, T. Zhang, J. Wang, and C. Zhang are with School of Instrument Science and Engineering, Southeast University, and Key Laboratory of Micro-Inertial Instrument and Advanced Navigation Technology, Ministry of Education, Southeast University, Nanjing 210096, China (e-mail: 230198704@seu.edu.cn; 101011356 @seu.edu.cn; 230169708@seu.edu.cn; 220193339@seu.edu.cn).

H.S. Shin is with the Institute of Aerospace Sciences, SATM, Cranfifield University, MK43 OAL Cranfifield, U.K. (e-mail: h.shin @cranfifield.ac.uk).

\section{INTRODUCTION}

A UTONOMOUS Underwater Vehicles (AUVs) can carry out comprehensive exploration of marine resources and other operating in dangerous waters. They have been applied in civil and military applications, such as oil resources surveys, submarine pipeline surveys, marine environment survey, and underwater equipment maintenance [1-3]. The information of accurate position, speed, and attitude provided by underwater navigation technology is the key to determine whether the AUV can reach the desired location accurately, complete the task successfully and return safely [4]. Unlike navigation systems of land or air carriers, the rapid attenuation of radio waves in the underwater environment makes the radio navigation system represented by the Global Navigation Satellite System (GNSS) no longer suitable for AUV [5]. Navigation has become one of the significant challenges in operations AUV.

At present, the common underwater navigation technologies mainly include inertial navigation, underwater acoustic positioning and navigation, and geophysical navigation [6-8]. Although inertial navigation is an autonomous navigation with strong concealment, its positioning error accumulates with time due to the error drift of inertial devices, which is not suitable for long-distance navigation $[9,10]$. Acoustic navigation (ultra-short baselines, short baselines, and long baselines) can provide the location information and has the advantages of high precision and no accumulated error. Nonetheless it requires the placement of transponders or signal sources underwater in advance, which is not suitable for applications such as ocean voyages [11]. Geophysical navigation technologies are based on the physical characteristics of the earth, mainly including terrain matching, geomagnetic matching and gravity matching, with the characteristics of strong autonomy, good concealment, and unrestricted by area and time [12-14]. The Terrain-Aided Navigation (TAN) algorithms have been reported to be successful in many applications. In [15], a novel algorithm combined with the terrain contour matching (TERCOM) algorithm and particle filter is proposed, which is reliable and achieves relatively high positioning accuracy in the case of large initial position errors and large altimeter measurement noises. Xiaojiao Ma [16] introduces the basic principle, composition and technologic application of Geomagnetic Aided Inertial Navigation System (GAINS). Gravity matching algorithm is a crucial technique of gravity aided navigation for underwater vehicles. Bo Wang [17] proposes a vector matching algorithm considering the correlation between adjacent sample points of INS output position. The single point matching algorithm used in the vector matching algorithm is based on 
particle filter, which is robust to the changes of gravity anomaly in the matching areas, with more accurate and reliable matching results. A matching algorithm combined with an iterated closest contour point (ICCP) algorithm and a point mass filter (PMF) is proposed in [18], which is better than that of the conventional ICCP algorithm and achieves more reliable result than the PMF. However, these navigation technologies strongly depend on the priori maps, and the problem is the accuracy and completeness of the maps are difficult to be ensured for various reasons.

In recent years, numerous studies have shown that many creatures on earth can locate and navigate according to the information of the earth's magnetic field [19]. Wilschko's work on the eurasian robin found that birds can use the vector direction and inclination of the magnetic field to orient themselves [20]. In [21], Lohmann found that the initial offshore migration of hatchling turtles can be accomplished with a suite of straightforward orientation steps involving visual cues, wave cues, and a magnetic compass sense. As turtles mature, they gain experience with geographic patterns of magnetic variation and learn to find goals based on magnetic positional information. Salmons can detect geomagnetic information, making the migratory route more predictable and facilitating movement into favorable oceanic regions [22]. Boles and Lohmann [23] carried out a series of experiments and found that lobsters can effectively use geomagnetic information to complete homing. During the past two decades, tremendous progress has been made in unraveling the mechanisms that underlie orientation and navigation in creatures.

The above studies show that many animals can effectively use geomagnetic information for navigation, proving that the GF is a reliable information source for long-distance movement of animals. It is less likely to store complete geomagnetic maps in their brains, which provides a biological basis for navigation without prior geomagnetic maps. At the same time, there is almost a one-to-one correlation between the GF vector and every point in near-earth space, which provides a sufficient theoretical basis for geomagnetic navigation. Besides, geomagnetic navigation is passive guidance with the characteristics of strong concealment, anti-interference, the error does not accumulate over time, and magnetic sensors are small in size and low in power consumption. Therefore, bionic geomagnetic navigation has gradually attracted significant attention.

As the name implies, bionic geomagnetic navigation is to simulate the process of animals' long-distance migration. Without the prior geomagnetic map, the destination can be reached by autonomous search through real-time sensing of geomagnetic information [23]. From the perspective of bionic navigation, the GF is a multivariate field containing multiple geomagnetic feature quantities, each of which has its own change law. Thus, bionic geomagnetic navigation can be summarized as a search for the geomagnetic multi-parameter and multi-targets when geomagnetic elements change law is unknown.

To address this problem, there have been different bionic geomagnetic navigation strategies are proposed in literature. References [24] and [25] introduced EKF and KF algorithms to find shortcuts in geomagnetic space based on the analysis of long-distance migration characteristics of animals such as pigeons and turtles using geomagnetic information. The simulation results show that geomagnetic navigation can still be achieved in various interference environments without the help of pre-stored geomagnetic and geographic data. However, the performance of the method is poor while passing through the geomagnetic anomaly areas. The research works have confirmed the feasibility of the underwater geomagnetic navigation, but navigation strategy in geomagnetic anomaly areas and navigation trajectory need to be further optimized. A long-distance geomagnetic navigation method based on model predictive control was proposed in [26], which calculates the optimal control sequence according to magnetic declination and magnetic inclination. However, the actual magnetic anomaly areas are often more complex and cannot be treated as a constant. The magnetic intensity of the weak abnormal area is less than $1 \mathrm{nT}$, and the magnetic intensity of the strong abnormal area could even reach several times of the main magnetic field. Similarly, inspired by animals' navigation behavior using geomagnetic information, Liu proposed a geomagnetic bionic navigation method based on timing evolutionary search strategy [27]. The objective function of geomagnetic multi-parameter is established based on the principle of animal magnetotropism. In the course of navigation, the evolutionary search mode is adopted to guide the carrier to approach the target point. However, the random navigation search method leads to the great fluctuation of the heading angle and zigzag path, which brings difficulties in engineering applications [28]. Meanwhile, the evaluation function is not accurate enough, which leads to the deviation of the navigation path from the optimal path, resulting in the waste of time and cost.

To reduce the randomness of carrier motion and improve the anti-interference of navigation algorithm, this article develops an enhanced evolutionary search algorithm based on the geomagnetic gradient. The bionic geomagnetic navigation is reduced to a multi-parameter and multi-objective search problem. The navigation path is searched based on the constraint relationship between the motion path and the geomagnetic parameters. With the aid of the geomagnetic gradient, the heading angle is predicted based on the principle of multi-parameter simultaneous convergence, which is used to constrain the sample space. Search the navigation path with constrained sample space can reduce invalid search and randomness, and consequently accelerate the convergence rate of magnetic elements. To search the optimal navigation path, the evaluation function in the evolutionary algorithm was improved to make the evaluation of each sample more accurate. Furthermore, the performance of the improved algorithm in the abnormal geomagnetic area is validated via simulations. Simulation results show that the algorithm developed can successively guide the carrier through the geomagnetic anomaly area with anti-interference capability.

The remainder of the article is organized as follows. Section II introduces the mathematical description of the GF and formulates the problem of bionic geomagnetic navigation. Section III presents an improved evolutionary algorithm to solve the convergence of geomagnetic multi-parameter about 
navigation for AUV. And the convergence performance of the new algorithm is analyzed and validated by simulation in Section IV. Conclusions are offered in Section V.

\section{FUNDAMENTALS}

\section{A. Description of the geomagnetic field}

The GF is a basic physical field of the earth. Theoretically, the geomagnetic vector at any point corresponds to its geographical position one by one in the near-earth space [29]. The GF is a weak magnetic field, and the strength of the GF is usually measured in nanotesla (nT). The average intensity on the ground is $5^{*} 10^{4} \mathrm{nT}$, and only about $7^{*} 10^{4} \mathrm{nT}$ at the two poles where the GF is strongest. The strength of the GF is mainly composed of three parts:

$$
\boldsymbol{B}(r, t)=\boldsymbol{B}_{m}(r, t)+\boldsymbol{B}_{e}(r, t)+\boldsymbol{B}_{d}(r, t)
$$

where $\boldsymbol{B}_{m}(r, t)$ is the main magnetic field, produced by large-scale electric currents in the liquid outer core of the earth consisting of highly conductive molten irons [30]. The change of the main magnetic field is very slow and the time cycle is measured on the scale of a thousand years. $\boldsymbol{B}_{e}(r, t)$ is geomagnetic anomaly field, generated from magnetized crustal rocks, which decaying with increasing height, and hardly changes with time. $\boldsymbol{B}_{d}(r, t)$ is called the disturbed magnetic field, originating in the magnetosphere and ionosphere. The size ranges from $5 \mathrm{nT}$ to $500 \mathrm{nT}$, which varies dramatically over time and is always associated with solar activity [31].

To describe the spatial distribution of the GF, the geomagnetic vector is decomposed into seven geomagnetic elements $\left(B_{F}, B_{H}, B_{x}, B_{y}, B_{z}, D, I\right)$ in the geographic coordinate system.

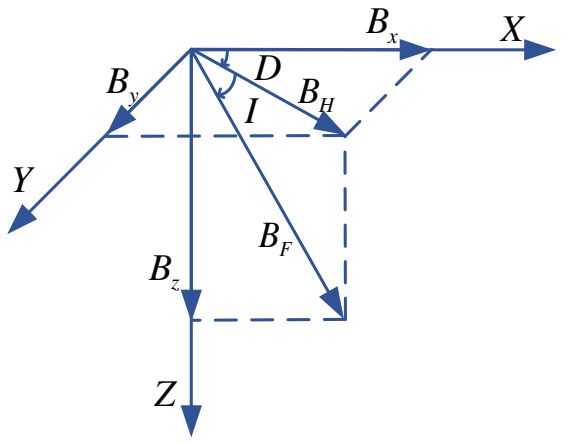

Fig. 1. Seven elements of the GF

As shown in Fig.1, the $\mathrm{x}$-axis is along with north, the y-axis is along with east, and the z-axis conforms to the right-hand rule. The total strength of the GF is denoted as $B_{F}$, and its projection on the horizontal plane is the horizontal component, denoted as $B_{H} . B_{x}$ and $B_{y}$ are the projections of $B_{H}$ in the north direction and east direction respectively, and $B_{z}$ is the projection of $B_{F}$ in the vertical direction, known as the vertical component. $\mathrm{D}$ is called the magnetic deviation, which is the angle between $B_{H}$ and $B_{x}$ in the horizontal plane. The angle between $B_{F}$ and the horizontal plane is called magnetic inclination, denoted as I. Given three of the seven elements, all of them can be calculated by (2).

$$
\left\{\begin{array}{l}
B_{x}=B_{F} \cos I \cos D \\
B_{y}=B_{F} \cos I \sin D \\
B_{z}=B_{F} \sin I \\
B_{F}=\sqrt{B_{x}^{2}+B_{y}^{2}+B_{z}^{2}} \\
B_{H}=B_{F} \cos I \\
I=\arccos \left(B_{H} / B_{F}\right) \\
D=\arccos \left(B_{x} / B_{H}\right)
\end{array}\right.
$$

The properties of the GF have been extensively investigated $[32,33]$. In general, the GF model described by IGRF12 (International Geomagnetic Reference Field 12) provides a realistic description of the global GF, including all geomagnetic elements. The resolution of the GF intensity is $1 \mathrm{nT}$, and the distance resolution is $0.01^{\circ}$ in latitude or longitude. This article studies in the geomagnetic environment constructed by IGRF12, rather than measuring the real GF. As the basis of geomagnetic navigation, the uniqueness of the GF is discussed in [26].

\section{B. Problem description}

The GF is a mixture of multiple elements, and the corresponding relationship between multi-parameter characteristics and near-earth spatial location provides a reliable physical basis for bionic navigation. The geomagnetic elements at a position in space can be described as:

$$
\mathbf{B}=\left\{B_{1}, B_{2}, \cdots, B_{n}\right\}
$$

where $B_{1}, B_{2}, \cdots, B_{n}$ are part or all of the geomagnetic elements. The carrier navigation process is the parameter convergence process from the initial position to the target position. Hence, the objective function is established as shown in (4) to describe its convergence process and judge whether the destination is reached.

$$
f_{i, k}(B)=\left(B_{i, k}-B_{i, T}\right)^{2}
$$

where $B_{i, k}$ and $B_{i, T}$ are the ith geomagnetic elements at time $\mathrm{k}$ and destination, respectively. Considering the difference in magnitude and unit, the objective function is normalized to:

$$
F\left(\boldsymbol{B}_{k}\right)=\frac{1}{n} \sum_{i=1}^{n} \frac{f_{i, k}(B)}{\left(B_{i, 0}-B_{i, T}\right)^{2}}
$$

where $B_{i, 0}$ is the ith geomagnetic element at the initial position.

When the carrier arrives at the destination, theoretically, the objective function is 0 .

The errors between the current position and the destination can be assumed as the geomagnetic trend, while the searching process is terminated when the error converges to $\varepsilon$. This is expressed as:

$$
F\left(\mathbf{B}_{k}\right) \leq \varepsilon
$$

where $\varepsilon$ is a minimum quantity close to 0 , which is set according to the navigation accuracy.

From the point of view of biological magnetism, bionic geomagnetic navigation can be regarded as a search behavior under the stimulation of various geomagnetic parameters. The navigation path is a search result induced by the variation trend 
of multiple geomagnetic parameters. There is a strong restrictive relationship between geomagnetic parameters and navigation path. Therefore, the convergence relation of the geomagnetic parameters in the course of navigation is established based on the geomagnetic trend sensitivity to the geomagnetic parameters at the destination.

\section{Algorithm}

To imitate the animals' long-distance homing, we assume two simple preconditions. First, the geomagnetic elements of the destination are known before navigation, as the reference to judge if the carrier have approach the destination. Second, the geomagnetic elements at any position can be obtained by the geomagnetic sensors in real-time.

First of all, the motion model of the carrier is established. The carrier can be regarded as a mass point in the navigation process based on GF, and its motion model can be expressed as:

$$
\left\{\begin{array}{l}
x_{k+1}=x_{k}+\cos \theta_{k} \cdot L \\
y_{k+1}=y_{k}+\sin \theta_{k} \cdot L
\end{array}\right.
$$

where $\left(x_{k}, y_{k}\right)$ represents the carrier position at time k. $\theta_{k}$ is the heading angle at time $\mathrm{k}, L$ is the movement step of the carrier. As can be seen from the above motion model, the heading angle and the movement step length are the inputs of the motion model to drive the carrier close to the target position. Among them, the heading angle, which determines the movement direction of the carrier, is the key factor. Thus, the multi-parameter search process in geomagnetic space can be transformed into solving the carrier heading angle. Meanwhile, it can be seen from (5) that the search process of multiple target geomagnetic parameters in geomagnetic space is essentially a multi-objective optimization problem of peak function.

The evolutionary strategy is an optimization method for multi-objective problems proposed by I. Rechenberg and $\mathrm{H}$. Sehwefel, who draw lessons from the idea of survival of the fittest in biological evolution. In the search process of evolutionary algorithm, it simulates the evolutionary law of nature and keeps excellent samples while constantly evolving and updating the population. The global optimal solution or approximate optimal solution is obtained while maintaining reasonable population diversity. Group search strategy and information exchange among individuals are the main features of evolutionary algorithms. The advantage of this algorithm is that the search process is not easy to fall into local optimum. Moreover, it adopts the natural evolution mechanism to represent the complex phenomenon, which can solve the difficult problems quickly and reliably. In addition, it is easy to be introduced into the existing models, and has strong scalability. Hence, this article adopts the evolutionary algorithm to solve the problem of multi-objective convergence and search the navigation path.

Combining with the navigation problem, the heading angle is selected as the population sample, which represents the search solution space of the multi-objective problem. Based on the constraint relationship between the navigation path and geomagnetic parameters, an evaluation function is established to perform posterior evaluation on the currently executed sample to update the population. As the population evolves, carrier motion parameters gradually evolve in the right direction, guiding the carrier toward the destination until the completion of navigation.

In the evolutionary algorithm, however, the probability of each sample being selected is the same. The trend of navigation search is determined by the proportion of the same individual. Samples in the population are often randomly selected in the absence of prior information. Only through continuous trial and error and adjustment, the population can converge to the target solution. Therefore, the randomness of carrier motion is inevitable in the initial navigation phase, which impedes practical applications. In order to reduce the randomness in the motion process, an evolutionary search algorithm based on gradient information is proposed. Fig.2 shows the path search strategy in the evolutionary algorithm.

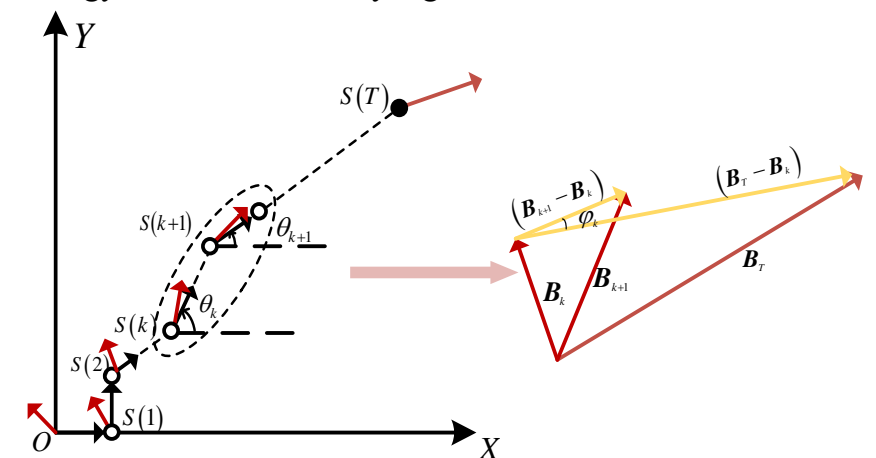

Fig. 2 Schematic of the navigation search strategy

In Fig.2, $\boldsymbol{B}_{k}$ denotes the geomagnetic vector of current position at time $\mathrm{k}, \boldsymbol{B}_{T}$ denotes the geomagnetic vector of the destination, $S(k)=\left(\begin{array}{ll}x_{k} & y_{k}\end{array}\right)$ is the carrier position at time $\mathrm{k}$, and $\varphi_{k}$ is the angle between vectors of $\left(\boldsymbol{B}_{k+1}-\boldsymbol{B}_{k}\right)$ and $\left(\boldsymbol{B}_{T}-\boldsymbol{B}_{k}\right)$.

To avoid the disordered search and make the navigation path close to the shortest path, the constraint relationship between the navigation path and geomagnetic parameters is established as shown in Fig.2. The difference of magnetic parameters between the destination and the current position is taken as the target vector in movement process. In the procedure of path search, it should be satisfied that the magnetic parameter difference vector of between adjacent positions tracks the target vector. Thus, the carrier trajectory is constrained according to (8).

$$
\left(\boldsymbol{B}_{k+1}-\boldsymbol{B}_{k}\right) / /\left(\boldsymbol{B}_{T}-\boldsymbol{B}_{k}\right)
$$

Projecting vectors $\left(\boldsymbol{B}_{k+1}-\boldsymbol{B}_{k}\right)$ and $\left(\boldsymbol{B}_{T}-\boldsymbol{B}_{k}\right)$ into the geographical coordinate system respectively, the following results can be obtained.

$$
\frac{B_{i, k+1}-B_{i, k}}{B_{i, T}-B_{i, k}}=\frac{B_{j, k+1}-B_{j, k}}{B_{j, T}-B_{j, k}}
$$

Therefore, if different geomagnetic elements $B_{i, k}(i=1,2, \cdots n)$ keep the same ratio close to the target geomagnetic elements, invalid searches can be avoided and the carrier can reach the destination as soon as possible.

To represent the relationship between geomagnetic elements at two adjacent moments, geomagnetic gradient information is introduced [35]. 


$$
\left\{\begin{array}{l}
B_{i, k+1}=B_{i, k}+g_{B_{i, k}, x} \cdot \cos \theta_{k}+g_{B_{i, k}, y} \cdot \sin \theta_{k} \\
B_{j, k+1}=B_{j, k}+g_{B_{j, k}, x} \cdot \cos \theta_{k}+g_{B_{j, k}, y} \cdot \sin \theta_{k}
\end{array}\right.
$$

where $g_{B_{i, k} x}, g_{B_{i, k} y}, g_{B_{j, k} x}, g_{B_{j, k} x}$ are the gradients of $B_{i, k}$ and $B_{j, k}$. By substituting (10) into (9), the heading angle can be obtained:

$$
\lambda_{k}=\arctan \left(\frac{\left(B_{i, k}-B_{i, T}\right) \cdot g_{B_{j, k} x}-\left(B_{j, k}-B_{j, T}\right) \cdot g_{B_{i, k} x}}{\left(B_{j, k}-B_{j, T}\right) \cdot g_{B_{i, k} y}-\left(B_{i, k}-B_{i, T}\right) \cdot g_{B_{j, k} y}}\right)
$$

For the convenience of expression, the predicted heading angle is denoted as $\lambda_{k}$. The gradients are obtained by the simple decomposition of the changes of geomagnetism elements between two successive sampling locations in the east and north directions [26]. Thus, the heading angles at step 1 and step 2 are set as $0^{\circ}$ and $90^{\circ}$ respectively, and the geomagnetic gradient was calculated through the geomagnetic elements measured by magnetic sensors.

$$
\begin{aligned}
& g_{B_{i, 2} x}=\left(B_{i, 1}-B_{i, 0}\right) / \mathrm{L} \\
& g_{B_{i, 2} y}=\left(B_{i, 2}-B_{i, 1}\right) / \mathrm{L} \\
& g_{B_{j, 2} x}=\left(B_{j, 1}-B_{j, 0}\right) / \mathrm{L} \\
& g_{B_{j, 2} y}=\left(B_{j, 2}-B_{j, 1}\right) / \mathrm{L}
\end{aligned}
$$

The heading angle can be predicted according to the above method. However, as the calculation of the gradient is not accurate, the error of the predicted heading angle is inevitable, which reduces the navigation accuracy. More importantly, the prediction method has poor anti-interference and is prone to be trapped in the geomagnetic anomaly area, resulting in navigation failure. Hence, an evolutionary search algorithm based gradient information method is proposed. The evolutionary algorithm is a heuristic search algorithm that simulates the process of biological evolution in nature. In the evolutionary algorithm, the carrier heading angle is taken as the population sample. The probability model of each sample can be used to simulate the trending movement behavior to construct a trending movement system with self-organizing ability.

Although there are errors in the prediction of heading angle based on the geomagnetic gradient, it provides an evolutionary trend for the population, which greatly reduces the randomness of the navigation path search. Therefore, the sample population is constrained based on the predicted heading angle in the proposed algorithm to reduce invalid search. Furthermore, a novel evaluation method is proposed in terms of the search strategy shown in Fig.3, to achieve a more accurate evaluation of the sample. In the process of sample updating, good sample individuals are retained to improve the efficiency of navigation path search. The steps and procedures for the algorithm are presented as follows.

Step 1: Sample initialization

Different from [28], the population no longer contains all the heading angle information. Instead, the population is constrained based on the predicted heading angle. Before that, it is necessary to evaluate the predicted heading angle. It is considered that the predicted heading angle is effective when certain requirements are met. The evaluation criteria will be explored in more detail below. To avoid contingency, the above prediction process is repeated many times. The optimal prediction result is selected to constrain the population. Through the discrete sampling of the heading angle, the set of navigation search schemes can be expressed as:

$$
\left\{\begin{array}{l}
\theta=\left\{\theta_{1}, \theta_{2}, \ldots, \theta_{N}\right\} \\
\theta_{i}=D_{\theta} \times R_{i} \\
\mathrm{R}_{i} \in\left[\left(\lambda_{o}-\beta\right) / \mathrm{D}_{\theta},\left(\lambda_{o}+\beta\right) / \mathrm{D}_{\theta}\right]
\end{array}\right.
$$

where $\mathrm{N}$ is population size, $D_{\theta}$ is sampling interval. $\beta$ is the threshold to constrain sample space, determined by the predicted heading angle. $\lambda_{o}$ is the optimal prediction result. $\mathrm{R}_{i}$ is a random integer within $\left[\left(\lambda_{o}-\beta\right) / \mathrm{D}_{\theta},\left(\lambda_{o}+\beta\right) / \mathrm{D}_{\theta}\right]$.

Step 2: Navigation termination condition

According to the objective function mentioned in Section II to determine whether the carrier has reached its destination. If so, the navigation process ends. Otherwise, continue with step 3.

Step 3: Sample selection

The sample is selected from the sample population in a non-preference manner and the probability of each sample being selected is the same. Thus, the probability of the heading angle being selected depends on its proportion of the same sample. At time $\mathrm{k}$, the probability of population sample $\theta_{i}$ being selected is:

$$
p\left(\theta_{i}, \mathrm{k}\right)=\frac{\sum_{j=1}^{N} \delta_{j}\left(\theta_{i}=\theta_{j}\right)}{N}, \delta_{j}\left(\theta_{i}=\theta_{j}\right)=\left\{\begin{array}{lc}
1, & \theta_{i}=\theta_{j} \\
0, & \text { else }
\end{array}\right.
$$

Step 4: Sample evaluation

In the natural environment of natural selection and survival of the fittest, each individual's adaptability to the environment affects its probability of reproduction. The evolutionary algorithm evaluates each sample by fitness function, and the individuals with high evaluation can survive, while those with low evaluation are eliminated easily, which drives the evolution of the population. Therefore, the establishment of sample evaluation mechanism is the key operator in the evolutionary algorithms, which directly determines the success or failure navigation. In the existing evolutionary algorithm, the monotone decline of the objective function is taken as the evaluation criterion [27, 28]. If the objective function at step $\mathrm{k}+1$ is less than step $\mathrm{k}$, it is considered that the selected sample is better, and should be propagated; otherwise, it should be replaced.

To analyze the accuracy of the objective function for sample evaluation, the distribution characteristics of the objective function are shown in Fig. 3. Fig. 3 shows the distribution characteristics of the objective function based on the EMM with $\left(5^{\circ} \mathrm{N}, 5^{\circ} \mathrm{E}\right)$ as the starting point and $\left(10^{\circ} \mathrm{N}, 10^{\circ} \mathrm{E}\right)$ as the destination. It is obvious from Fig. 3 that the objective function decreases gradually as the carrier approaches the destination. The optimal navigation scheme satisfies the characteristic of monotonic decreasing of objective function. However, there are many feasible navigation schemes to satisfy the feature, as shown in Fig. 3, including some inferior navigation schemes, which make the navigation path deviate from the optimal path and even lead to navigation failure. This is because the monotone decreasing of the objective function is a necessary condition rather than a sufficient condition for navigation path 
search. Therefore, a new evaluation method is proposed to improve the performance of the algorithm. While completing the navigation task, it is necessary to improve navigation efficiency and reach the destination with a shorter path.

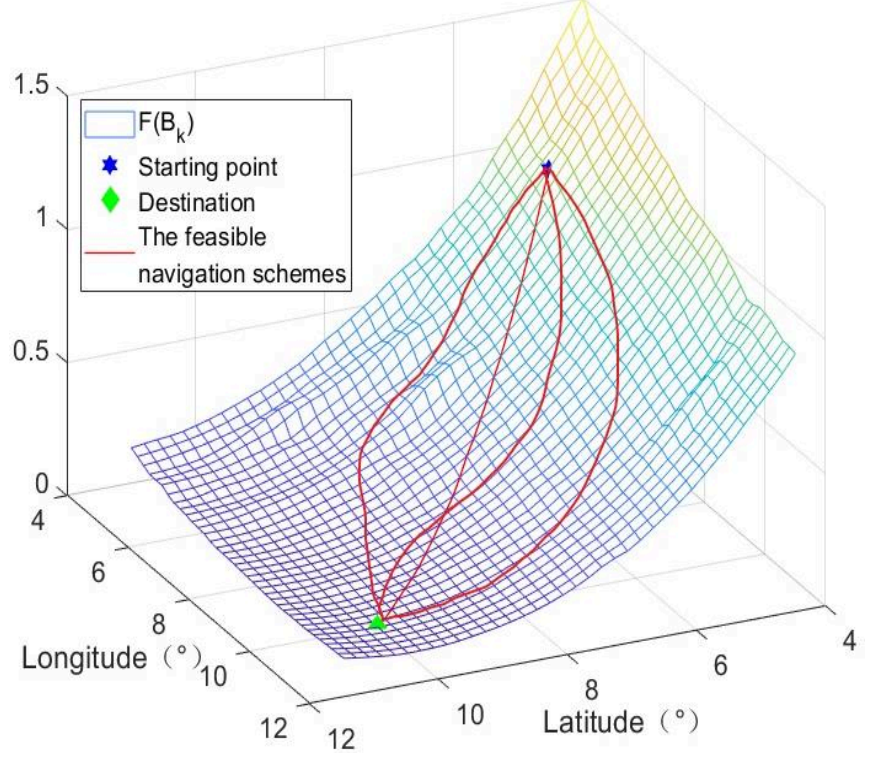

Fig. 3 The distribution characteristics of objective function

The above analysis shows that the search strategy in Fig.2 can avoid invalid search and improve the search efficiency. In this circumstance, angle $\varphi_{k}$ is zero or close to zero. Thus, the evaluation function can be established on the basis of the above principles, that is, the samples can be evaluated by observing the angle $\varphi_{k}$.

$$
\varphi_{k}=\arccos \left(\frac{\left(\boldsymbol{B}_{T}-\boldsymbol{B}_{k}\right) \cdot\left(\boldsymbol{B}_{k+1}-\boldsymbol{B}_{k}\right)}{\left|\boldsymbol{B}_{T}-\boldsymbol{B}_{k}\right| \cdot\left|\boldsymbol{B}_{k+1}-\boldsymbol{B}_{k}\right|}\right)
$$

Then, the heading angle predicted is considered valid when $\varphi_{k}$ is less than $15^{\circ}$, which is set by data statistics of multiple simulations.

Step 5: Sample update

Based on the above evaluation criteria, the updating rules of sample space are formulated.

$$
\left\{\begin{array}{cc}
5^{\circ}<\varphi_{k+1} \leq 15^{\circ} \& \varphi_{k+1} \leq \varphi_{k} & \text { propagate, } P_{p}=0.1 \\
0^{\circ}<\varphi_{k+1} \leq 5^{\circ} \& \varphi_{k+1} \leq \varphi_{k} & \text { propagate, } P_{p}=0.3 \\
\text { else } & \text { update }
\end{array}\right.
$$

where $\boldsymbol{P}_{p}$ denotes propagation ratio, which has a great influence on the convergence rate of the population. In the process of path searching, on the one hand, evolutionary algorithm should keep the good individuals and converge to the global optimal solution by the principle of survival of the fittest. On the other hand, it is necessary to keep the population diversity, expand the search space and avoid premature convergence. For different optimization problems, it is common practice to determine $\boldsymbol{P}_{p}$ through repeated experimental analysis.

Different from other optimization problems, in bionic navigation, the carrier is required to adjust the navigation search scheme after trial and error due to the sample posterior evaluation. Hence, the rapid convergence of population is conducive to reduce the randomness of the carrier movement. As shown in (15), different reproduction rates are set to accelerate population convergence on the basis of sample evaluation. $P_{p}$ is set by data statistics of multiple simulations. When the condition $\left(\varphi_{k+1} \leq \varphi_{k}\right) \&\left(\varphi_{k+1}<15^{\circ}\right)$ is satisfied, it is considered that the carrier approaches the destination, and the sample is an excellent search scheme. Furthermore, other individuals were randomly selected according to the propagation ratio and replaced by the excellent sample. Otherwise, the carrier is considered to be far away from the destination, and the sample would lead to an inferior search path, which is replaced by a randomly generated sample. With the search of the navigation path, the sample space also tends to evolve in the correct direction. Besides, it's important to note that the randomly generated samples are also constrained by (13).

In the course of navigation search, the motion path is not only the result of navigation search but also the multi-parameter change caused by path search continuously induces carrier to the destination. The workflow of the improved evolutionary algorithm is shown in Fig.4.

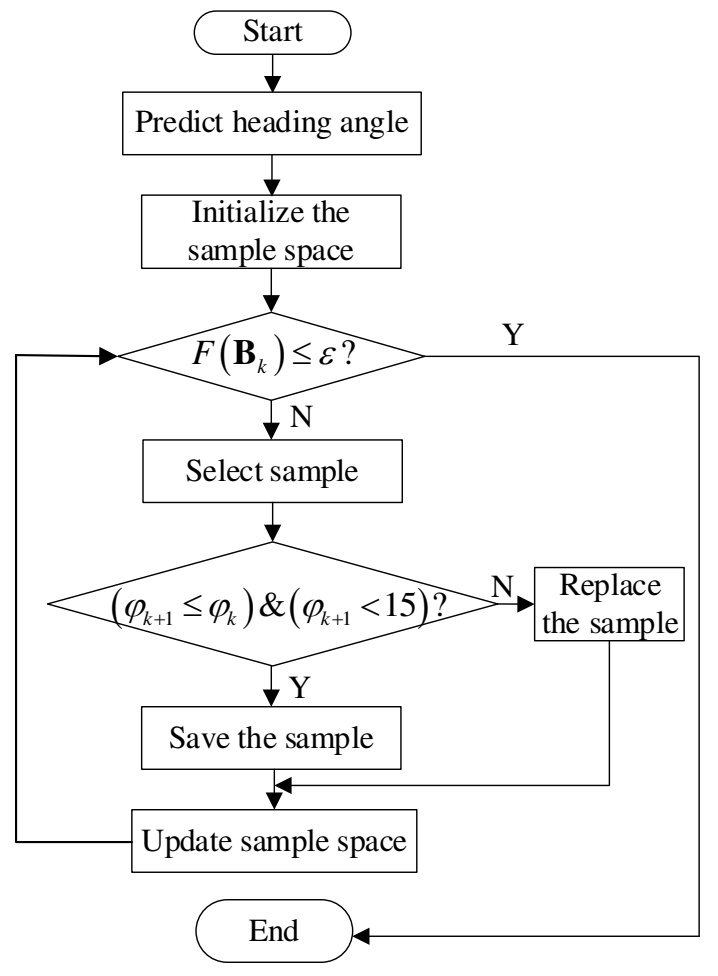

Fig. 4 Flowchart of the improved algorithm

\section{SIMULATION}

This section performs the simulations to verify the performance of the improved algorithm, in which the geomagnetic elements are retrieved from the Enhanced Magnetic Model (EMM). The standard World Magnetic Model uses a spherical harmonic representation to degree and order 12, resolving the magnetic field at $3000 \mathrm{~km}$ wavelength. In contrast, the EMM extends to degree and order 790, resolving magnetic anomalies down to $51 \mathrm{~km}$ wavelength. The higher resolution of the EMM results in significantly improved pointing accuracy. 
At the beginning of navigation, the heading angles are set as $0^{\circ}$ and $90^{\circ}$ respectively, and the geomagnetic gradient is calculated from (12). Then, the heading angle is predicted with the gradient information. In order to make the prediction more accurate, the selected geomagnetic characteristics should have distinct statistical characteristics and contain enough characteristic information in the navigation search area. Reference [36] measured the similarity of the seven geomagnetic characteristics by Euclidean distance. In the navigation area of the simulations, $B_{y}$ and $B_{z}$ have the lowest similarity. Thus, $B_{y}$ and $B_{z}$ are selected to predict heading angle by (11). $B_{x}, B_{y}$ and $B_{z}$ are selected to search the navigation path, which can directly describe the vector characteristics of geomagnetism. Meanwhile, the simulation parameters are set as shown in Table. 1, in which the settings of relevant parameters in the evolutionary algorithm are changed for different optimization problems. The influence of each parameter on the algorithm performance and the optimal selection problem can be referred to [37].

In the improved algorithm, if the constraint threshold of population sample is too large, the randomness of carrier will increase, otherwise, the robustness of evolutionary algorithm will be reduced. Hence, the population constraint threshold is set by data statistics of multiple simulations. In order to get a better navigation accuracy, the step size of the carrier is set to 3 nmile when the objective function $F\left(\boldsymbol{B}^{k}\right)$ is not less than 0.005 , otherwise, the step size is set to 1.5 nmile. The parameter of triggering iteration step reduction can be adjusted according to the setting of navigation termination condition. When the objective function $F\left(\boldsymbol{B}^{k}\right)$ is less than 0.0001, it is considered that the carrier has reached the destination. The setting of navigation termination condition is related to the actual navigation accuracy requirements.

To prove the effectiveness of the improved algorithm, in addition, simulations are carried out for the navigation algorithm before and after improvement. The sample space range in the navigation algorithm before improvement is 0 to $360^{\circ}$, the sample interval is $30^{\circ}$, and the monotone decline of the objective function as shown in (5) is taken as the evaluation criterion. When $F\left(\boldsymbol{B}_{k}\right)$ is smaller than $F\left(\boldsymbol{B}_{k-1}\right)$, the current sample is considered to be a good sample, then the reproduction operation is carried out and $P_{p}=0.1$, otherwise, the sample is removed.

TABLE 1. SIMULATION PARAMETERS

\begin{tabular}{c|c|c}
\hline & $\begin{array}{c}\text { The improved } \\
\text { algorithm }\end{array}$ & $\begin{array}{c}\text { The original } \\
\text { algorithm }\end{array}$ \\
\hline Population size $(\mathrm{N})$ & 35 & 35 \\
\hline Sampling interval $\left(D_{\theta}\right)$ & $1^{\circ}$ & $30^{\circ}$ \\
\hline $\begin{array}{c}\text { Threshold for } \\
\text { ending navigation }(\varepsilon)\end{array}$ & 0.0001 & 0.0001 \\
\hline $\begin{array}{c}\text { Threshold for constraining } \\
\text { sample space }(\beta)\end{array}$ & $40^{\circ}$ & $/$ \\
\hline Evaluation function & $\varphi_{k}$ & $F\left(\boldsymbol{B}_{k}\right)$ \\
\hline
\end{tabular}

\section{A. Simulation Without Interferences}

In the ideal conditions, the geomagnetic anomaly area is not taken into account. In the simulation, the navigation process is started from $\left(5^{\circ} \mathrm{N}, 5^{\circ} \mathrm{E}\right)$, where geomagnetic components $\left(B_{x}, B_{y}, B_{z}\right)$ are $(31349.8 \mathrm{nT},-985.1 \mathrm{nT},-9893.7 \mathrm{nT})$. The destination is $\left(10^{\circ} \mathrm{N}, 10^{\circ} \mathrm{E}\right)$, where geomagnetic three components are (34052.4 nT, $151.3 \mathrm{nT},-2213.1 \mathrm{nT})$. The improved algorithm is simulated following the flowchart given in Fig.4. The carrier moves along the $\mathrm{X}$-axis and $\mathrm{Y}$-axis respectively, to calculate the gradient information. Then, following to (11) and (15), the heading angle is predicted and used as an input to drive the carrier to the next position, and then the posterior evaluation of the sample is performed. To avoid contingency, repeat the above process three times, that is, the first three motion direction of carrier is determined by the predicted heading angle. The results are shown in Table 2.

TABLE 2. PREDICTION AND EVALUATION RESULTS OF HEADING ANGLE

\begin{tabular}{c|c|c|c}
\hline Heading angle $\left(\lambda /^{\circ}\right)$ & 45.3473 & 45.3383 & 45.3216 \\
\hline Evaluation results $\left.\varphi /^{\circ}\right)$ & 8.5303 & 8.6023 & 8.6234 \\
\hline
\end{tabular}

When the evaluation result is less than $15^{\circ}$, the predicted value is considered valid. According to the constraint principle mentioned in Section III, the sample space initialized is: $\theta_{i} \in\left[5^{\circ}, 85^{\circ}\right]$.

The simulation results are shown in Fig. 4. Trajectory 1 is the search result of the improved algorithm with the destination $\left(10.0044^{\circ} \mathrm{N}, 9.9631^{\circ} \mathrm{E}\right)$, and trajectory 2 is the search path of the original algorithm, ending the navigation with $\left(9.9904^{\circ} \mathrm{N}\right.$, $\left.10.0577^{\circ} \mathrm{E}\right)$.

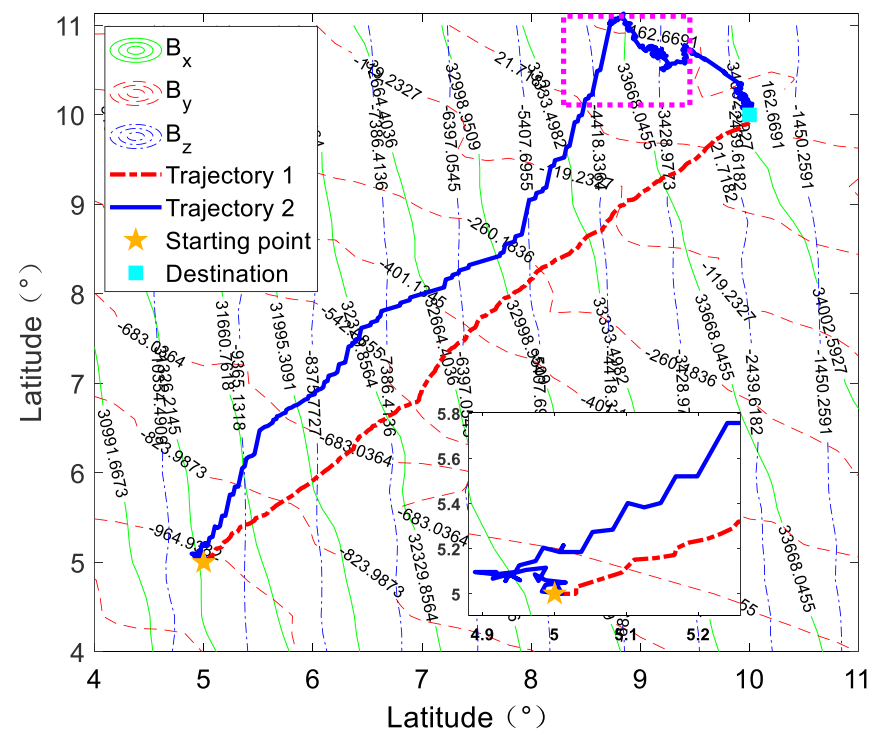

Fig. 5 Simulation of geomagnetic navigation without interference

As expected, trajectory 1 takes a shortcut in navigation search and is much flatter than the path searching by the algorithm before improvement. As seen in Fig. 5, there are some fluctuations in both paths at the initial navigation stage, which conforms to the feature of random search without prior information. However, due to the constraint of sample space in trajectory 1 , the fluctuation is far less than trajectory 2 , and the randomness is greatly improved. Compared to trajectory 2, trajectory 1 greatly shortens the navigation path, validating the effectiveness and correctness of improved evaluation function.

To illustrate the effectiveness of the improved algorithm, the convergence states of magnetic elements are shown in Fig. 6-7, 
where Fig. 6 is the convergence curve corresponding to trajectory 1, and Fig. 7 is the convergence curve of trajectory 2 .

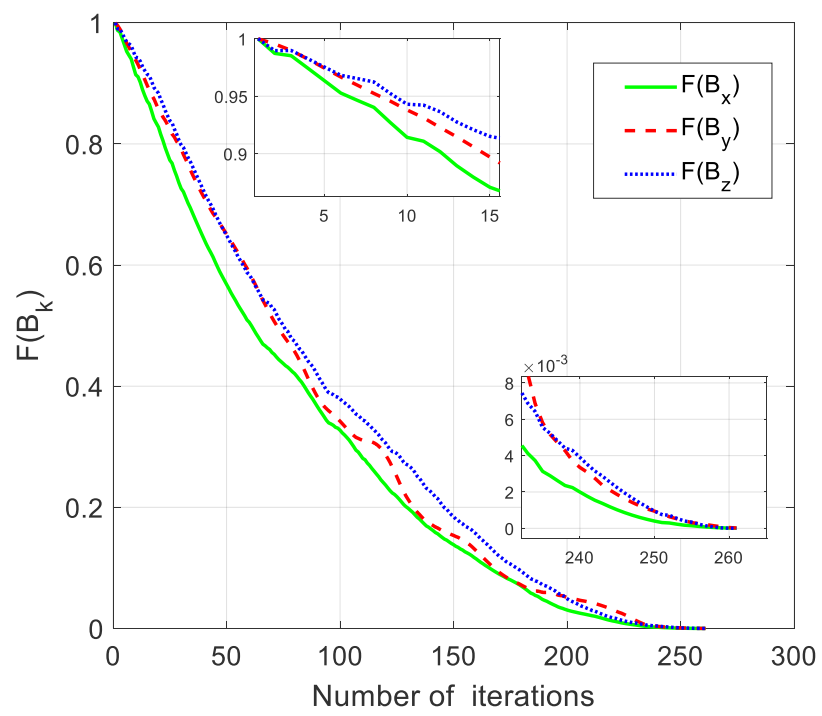

Fig. 6 The convergence curve of geomagnetic elements in trajectory 1

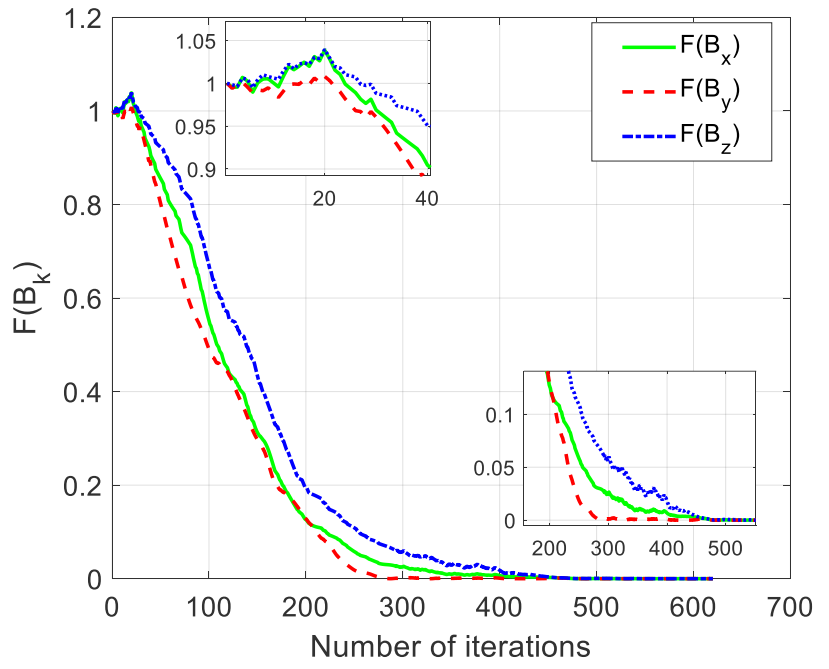

Fig. 7 The convergence curve of geomagnetic elements in trajectory 2

Three pieces of information can be obtained from the convergence curve of the objective function, which are the number of iterations, the smoothness of the convergence curves, and the convergence speed of each geomagnetic parameter.

Since the step size of the carrier is the same as shown in (7), the length of the search path is determined by the number of iteration steps. As can be seen from Fig. 6 and Fig. 7, trajectory 1 can reach the destination through 261 iterations, while trajectory 2 needs 620 iterations. Consequently, the improved method can reach the destination with higher search efficiency, which is consistent with the results in Fig. 5.

As the geomagnetism changes slowly, the zigzag search path will lead to the convergence curve to be unstable. The sample population in the original method contains all the feasible solutions. In each iteration, the fitness degree of selected individual is evaluated with respect to a given objective function. The highly fitted individuals have more opportunities to survive, whereas individuals who are less fitted will be eliminated. The sample population gradually converges to a better state after continuous trial and error adjustment. As shown in trajectory 2, however, the trial and error adjustment process directly leads to the randomness of path search. This is also the reason why the geomagnetic parameter objective function in the initial stage of navigation is greater than 1 as shown in Fig. 7.

In the improved method, the sample space is constrained based on the geomagnetic gradient information, which improves the search efficiency and reduces the randomness of path search. Moreover, the smoothness of the path is reflected in the convergence of the objective function. Just as the initial navigation stage of trajectory 2 , the fluctuation of the navigation path leads to the uneven convergence of the objective function as shown in Fig. 7. Meanwhile, the smoothness of the convergence curves corresponds to the flatness of trajectory 1 , compared with the convergence curves in Fig. 6.

From the constraint relationship between the navigation path and the geomagnetic parameters in Fig. 3, it can be seen that if the objective function converges along one of the geomagnetic parameters, that is, the convergence speed of each magnetic parameter is different, the search path will deviate from the destination. From Fig. 7, it can be observed that the convergence speed of the $F\left(B_{y}\right)$ is significantly faster than the other two parameters around 300th step, which corresponding to the large deviation of trajectory 2 as shown in the pink dotted box in Fig. 4. This is because in the original method, the monotone decline of the objective function is taken as the evaluation function, which is a sufficient condition for path search, but not a necessary condition, as shown in Fig. 3. The inaccurate evaluation function directly makes the inferior samples remain in the population, which makes trajectory 2 deviate from the destination. This article improves the evaluation function based on the relationship between the geomagnetic parameters and the navigation path to achieve the simultaneous convergence of the geomagnetic parameters as much as possible. As shown in Fig. 6, the uniform convergence of each parameter corresponds to a shorter navigation path (trajectory 1).

\section{B. Simulation with geomagnetic anomaly}

The geomagnetic anomaly field is caused by the uneven distribution of magnetic rocks in the earth's crust, which leads to the different sizes and intensity of the geomagnetism anomaly field. The strength of the weak abnormal field is less than $1 \mathrm{nT}$, while the strength of some strong abnormal fields can reach to several times of the main EMM. According to the superposition principle of magnetic field, the geomagnetic anomaly area changes the spatial distribution characteristics of normal GF parameters, which may form local extremum area and produce strong interference field. It is inevitable to encounter geomagnetic anomalies. Therefore, the performance of the improved algorithm in the geomagnetic anomaly area is analyzed by simulation.

The variation of geomagnetic anomaly field is complex, changeable and unpredictable. According to the influence of geomagnetic anomalies on navigation, complex geomagnetic anomalies can be divided into two categories: interference with geomagnetic features approaching the destination and interference with geomagnetic features far away from the 
destination. Therefore, the geomagnetic anomaly environment is constructed by superimposing EMM and multi-modal function in this simulation. The multi-peak function selected here is the PEAKS function in Matlab, and the geomagnetic anomaly area is $6^{\circ} \sim 8^{\circ} \mathrm{N}$ and $5.5^{\circ} \sim 7.5^{\circ}$ E. As shown in Fig. 8, the geomagnetic anomaly area contains the geomagnetic characteristics of approaching and far away from the destination respectively, and the intensity of geomagnetic anomaly is greater than the geomagnetic intensity of destination.

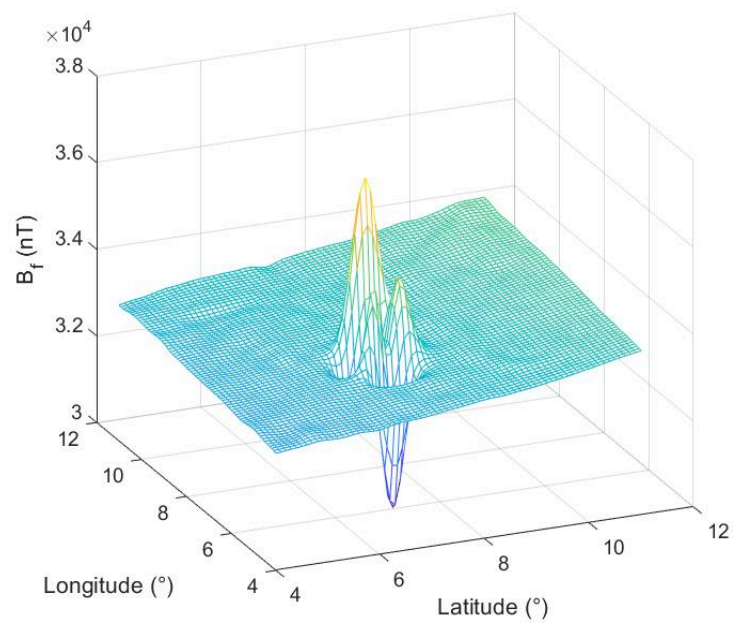

Fig. 8 Variation of geomagnetic strength in navigation area

The search for the navigation path is shown in Fig. 9, where trajectory 1 is the search path of the improved algorithm, trajectory 2 is the search path of the original algorithm. Trajectory 1 can reach the destination through 296 iterations. In trajectory 2 , the carrier enters the geomagnetic anomaly area after 50 iterations, but the carrier is still in the abnormal area when the iteration number has reached 700 times, resulting in navigation failure.

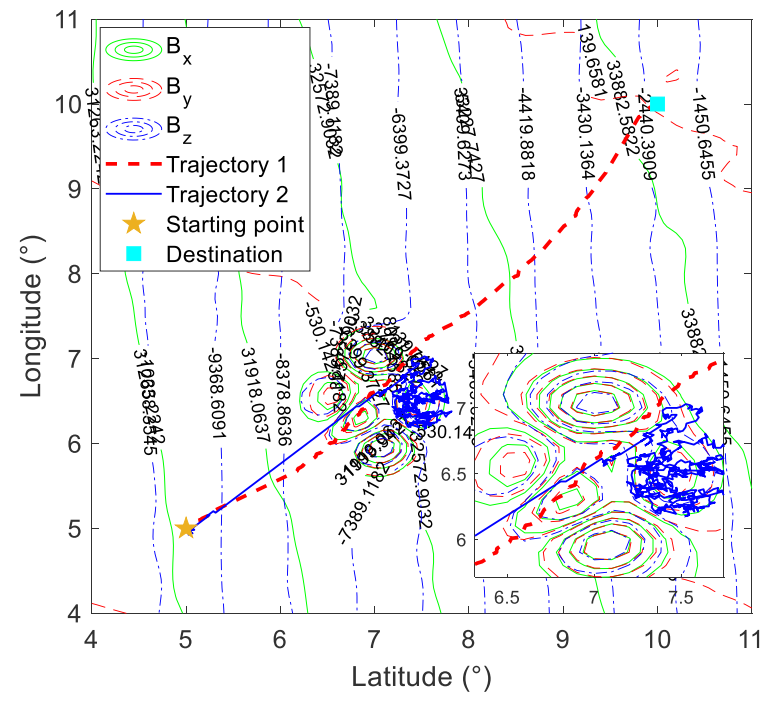

Fig. 9 Simulation of geomagnetic navigation with geomagnetic anomaly

It is obvious from Fig. 8 that the superposition of geomagnetic anomaly area and normal geomagnetic field results in the change of constraint relationship between geomagnetic parameters and navigation path. The objective function is no longer monotone decreasing. Therefore, in the original algorithm, the samples cannot be correctly evaluated, which makes the population no longer converge, and the carrier falls into chaotic search state. Instead of falling into the abnormal area, the improved algorithm overcomes the interference of the abnormal field and leaves the abnormal area. In the improved algorithm, the sample space is restricted based on the assistance of geomagnetic gradient, which greatly reduces the invalid search of the carrier, so that the carrier can leave the abnormal area. After the carrier goes out of the abnormal area of GF, the correct heading can be quickly re-searched to guide the carrier to the destination.

Similarly, the convergence states of objective function are shown in Fig. 10-11. As shown in Fig. 10, objective function no longer converges when passing through the abnormal area. It can be concluded that the constraint rule between navigation path and geomagnetic parameters is not applicable to the geomagnetic anomaly area. From the definition of the objective function shown in (5), it can be known that the dramatic decrease of $F\left(B_{x}\right)$ and $F\left(B_{z}\right)$ in Fig. 10 is due to the fact that the characteristics of the geomagnetic anomaly area passed by the carrier are closer to those of the destination. The increase of $F\left(B_{y}\right)$ is due to the greater difference between the geomagnetic characteristics of the anomaly area and that of the destination. It is concluded that the performance of the objective function varies with the characteristics of the geomagnetic anomaly. The objective function of trajectory 2 in Fig. 11 is no longer convergent due to the unordered searching. The objective function changes with the change of search path.

By comparing the convergence curves of Fig.10 and Fig.11, it can be found that the key to leaving the abnormal area is whether the objective function can break through the limitation of evaluation function. In the original algorithm, the samples are updated according to the original evaluation criteria. As the constraint relationship between geomagnetic parameters and the navigation path changes in the geomagnetic anomaly area, the evaluation criteria cannot accurately evaluate the samples, which makes the original algorithm fall into a disordered search state. However, the improved algorithm with the constrained sample space can overcome the change of the constraint relationship between the geomagnetic elements and the path, so as to get out of the abnormal area.

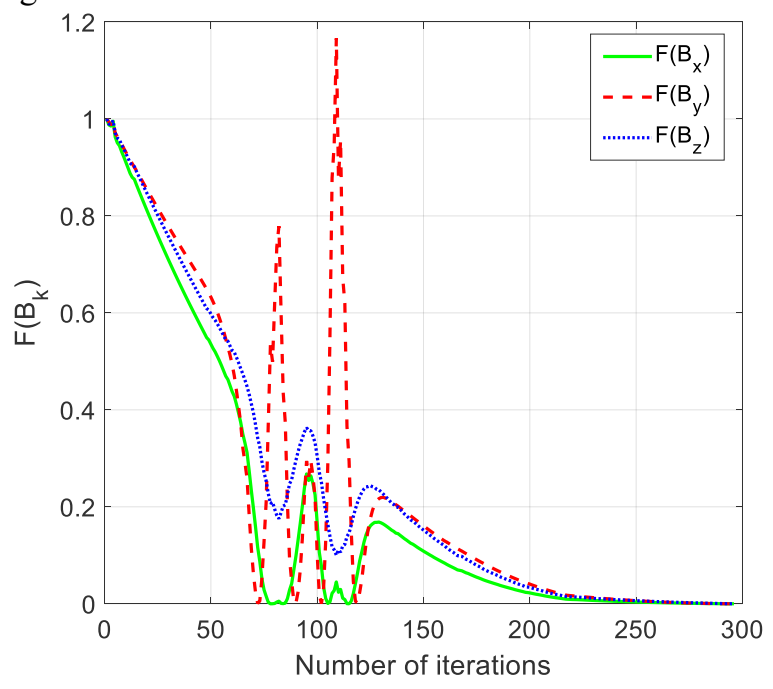

Fig. 10 The convergence curve of geomagnetic elements in trajectory 1 


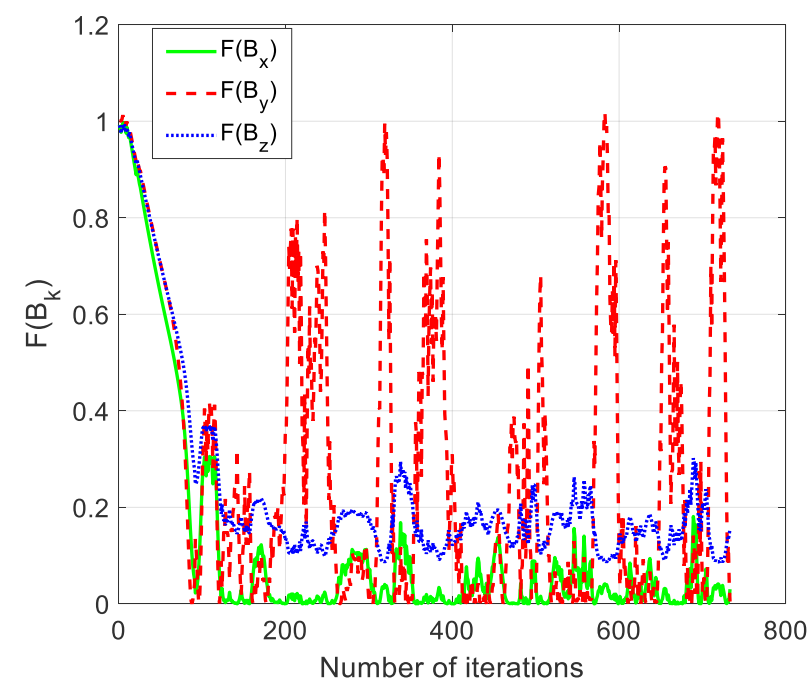

Fig. 11 The convergence curve of geomagnetic elements in trajectory 2

\section{Simulation with multi-destination navigation}

In practical applications, there may be multiple destinations to visit with varying missions. In the simulation, the feasibility and effectiveness of the multi-destinations are verified. In the simulation, the navigation is started from $\left(2^{\circ} \mathrm{N}, 2^{\circ} \mathrm{E}\right)$, and the destinations are $\left(3^{\circ} \mathrm{N}, 8^{\circ} \mathrm{E}\right),\left(8^{\circ} \mathrm{N}, 9^{\circ} \mathrm{E}\right)$, and $\left(7^{\circ} \mathrm{N}, 3^{\circ} \mathrm{E}\right)$, respectively. Then the carrier returns to the starting point. In simulation, the performances of the improved algorithm with the old one are compared, and simulation condition settings are shown in Table 1. The simulation results are shown in Fig. 12, where trajectory 1 is the search path of the improved algorithm, trajectory 2 is the search path of the original algorithm. Compared with trajectory 2 , trajectory 1 can visit each destination under a shortcut path during the navigation. Moreover, it's worth noting that navigation accuracy is related to the judgment condition of navigation termination and magnetic sensor performance. When the carrier arrives near the destination, the method can be combined with other navigation methods, such as the gravity-assisted method and terrain-based navigation techniques, to improve the navigation accuracy.

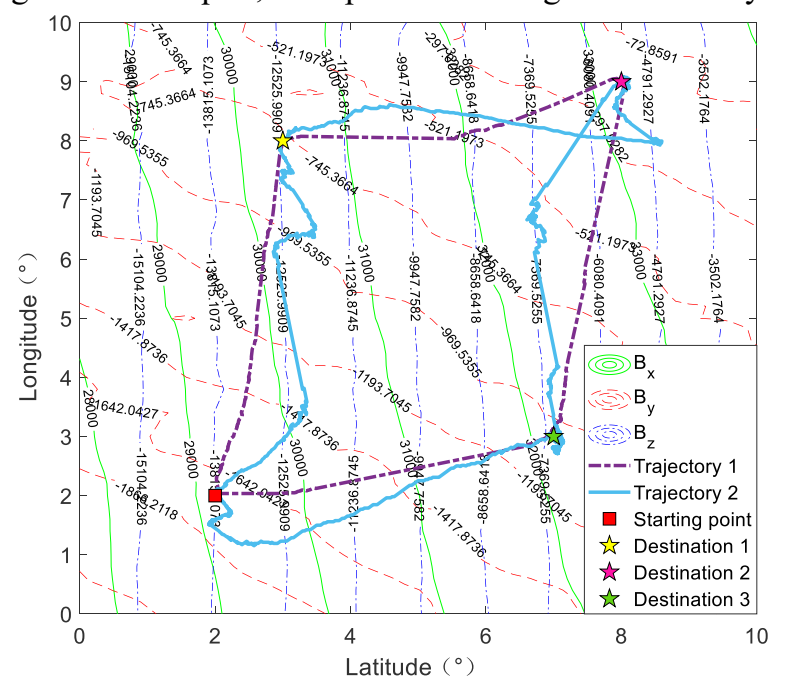

Fig. 12 Simulation with multi-destination navigation

In order to evaluate the performance of the search algorithm more accurately, it is very important to give a reasonable evaluation index. Since the step length of the carrier is the same in the algorithm before and after the improvement, the length of the path is determined by the number of iteration steps. Moreover, it can be seen from (7) that the search of the navigation path depends on the optimization solution of the heading angle $\theta_{k}$. Therefore, the statistical characteristics of the heading angle can reflect the straightness of the navigation path. In order to analyze the effectiveness of the improved algorithm, 200 simulation results are analyzed, including the average of iteration steps, average and variance of heading angle, as shown in Fig. 13. It should be noted that the target value of heading angle is calculated from the actual geographical location.

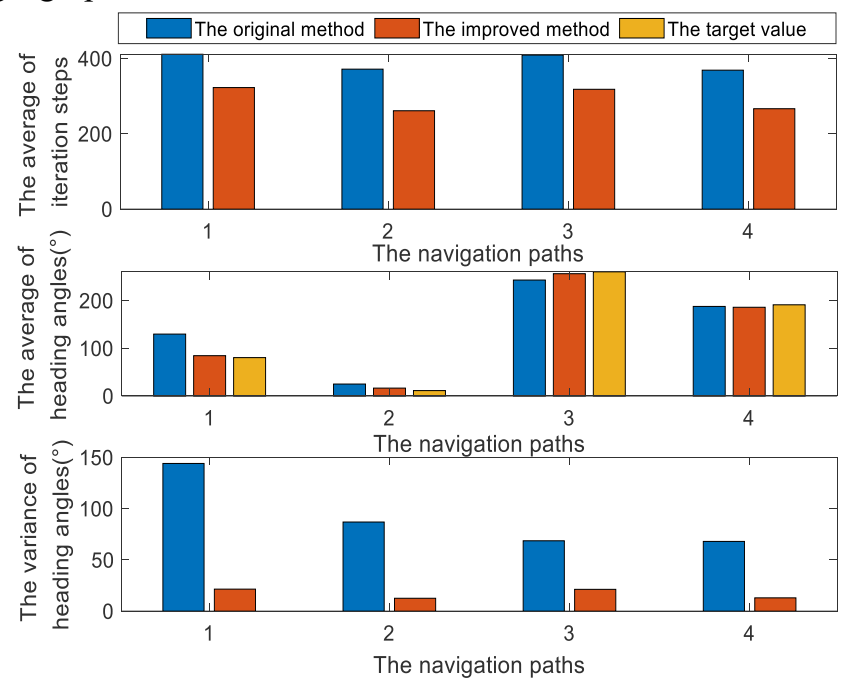

Fig. 13 The statistical chart of simulation results

It can be seen from Fig. 13 that the number of iteration steps of the improved method is smaller than that of the original method, and the number of iterative steps is reduced by $27.29 \%$ through quantitative analysis. From the average chart of the heading angle, moreover, it is can be known the optimized solution of the heading angle by the improved method is closer to the target value. The variance chart of the heading angle shows that the improved method has a smaller dispersion of the heading angle, which directly determines the stability of the navigation path. The improved method not only improves the efficiency of path search, but also greatly improves the stability of the path, which is more convenient for practical application.

In conclusion, in the absence of the priori geomagnetic information, the bionic navigation algorithm based on evolution strategy combines multi-objective solution with navigation motion to simulate biological magnetotaxis. In the course of navigation path search, the search of favorable navigation motion parameters got rid of the limitation that geomagnetic navigation must have a priori geomagnetic map. The effectiveness of the improved algorithm is verified by simulation.

\section{CONCLUSION}

This article proposed a geomagnetic gradient-assisted multi-objective evolutionary search algorithm for long-distance underwater navigation. Taking the geomagnetic elements of destination as the target, the efficient and fast path search is 
carried out in the absence of a priori geomagnetic information to realize long-distance geomagnetic autonomous navigation. The feasibility of the improved algorithm is verified based on the data retrieved from the EMM in real-time. Aiming at the problems of inefficient search and winding navigation path, the heading angle predicted by geomagnetic gradient information is used to constrain the sample space of the evolutionary algorithm. Meanwhile, the evaluation function is improved to enhance the search efficiency and anti-interference, to make the navigation path more reliable and accurate. The simulation results show that the proposed algorithm can successfully guide the carrier to the destination and has good performance under the interference of geomagnetic anomalies. Compared with the evolutionary method before improvement, the search efficiency of navigation path is greatly improved, which makes the navigation path more straight and convenient for practical application. It should be noted that navigation accuracy is related to navigation termination conditions and magnetic sensor performance. The slow change of geomagnetic information with geographic location determines that this method is more suitable for long-distance navigation applications. When the carrier is close to the destination, consider combining it with other navigation methods to obtain higher navigation accuracy. The research of bionic navigation is not only to reveal the behavior mechanism of birds, fishes and other organisms, but also to provide theoretical reference for AUV navigation application. It is possible to make the AUV navigation system have high autonomy and environmental adaptability. Although the algorithm has the ability to represent the motion behavior of AUV, it does not explore and reveal the internal mechanism deeply. In the future, it can continue to improve the algorithm or combine with other navigation methods to make AUV complete the navigation task more efficiently.

\section{REFERENCES}

[1] M. Bayat, N. Crasta, A. P. Aguiar and A. M. Pascoal, "Range-Based Underwater Vehicle Localization in the Presence of Unknown Ocean Currents: Theory and Experiments," IEEE Transactions on Control Systems Technology., vol. 24, no. 1, pp. 122-139, Jan. 2016, 10.1109/TCST.2015.2420636

[2] Wynn, R. B., Huvenne, V. A. I., Le Bas, T. P., Murton, B. J., Connelly, D. P., Bett, B. J., et al. "Autonomous underwater vehicles (AUVs): their past, present and future contributions to the advancement of marine geosciences," Mar. Geol., vol. 352, no. 1, pp. 451-468 Jun. 2014, 10.1016/j.margeo.2014.03.012.

[3] T. Maki, K. Asakawa, W. J. Kirkwood, "Guest Editorial Cutting edge autonomous underwater vehicle technology," IEEE Journal of Oceanic Engineering., vol. 43, no. 2, pp. 286-287, Apr. 2018.

[4] Y. Huang, Y. Zhang, Y. Zhao, "Review of Autonomous Undersea Vehicle Navigation Methods," Journal of Unmanned Undersea Systems, vol. 27, No. 3, pp. 232-253, Jun. 2017.

[5] P. Misra, B. P. Burke, and M. M. Pratt, "GPS performance in navigation,” Proc. IEEE, vol. 87, no. 1, pp. 65-85, Jan. 1999.

[6] J. C. Kinsey, R. M. Eustice, and L. L. Whitcomb, "A survey of underwater vehicle navigation: recent advances and new challenges," in Proc. IFAC Conf. MCMC, Lisbon, Portugal, Jan. 2006, pp. 1-12.

[7] Q. Li, Y. Ben, S. M. Naqvi, J. A. Neasham and J. A. Chambers, "Robust Student's t -Based Cooperative Navigation for Autonomous Underwater Vehicles," IEEE Transactions on Instrumentation and Measurement., vol. 67, no. 8, pp. 1762-1777, Aug. 2018.

[8] W. Li, W. Wu, J. Wang, and M. Wu, "A novel backtracking navigation scheme for Autonomous Underwater Vehicles," Measurement, vol. 47, pp. 496-504, Jan. 2014.
[9] X. Gong, J. Zhang and J. Fang, "A modified nonlinear two-filter smoothing for high-precision airborne integrated GPS and inertial navigation," IEEE Transactions on Instrumentation and Measurement, vol. 64, no. 12, pp. 3315-3322, Dec. 2015.

[10] J. Li, J. Fang, "Not Fully Overlapping Allan Variance and Total Variance for Inertial Sensor Stochastic Error Analysis," IEEE Transactions on Instrumentation and Measurement, vol. 62, no. 10, pp. 2659-2672, Oct. 2013.

[11] T. Zhang, L. Chen, Y. Xiong. "Underwater Positioning Algorithm Based on SINS/LBL Integrated System," IEEE Access., vol. 6, pp. 7157-7163, Jan. 2018.

[12] H. Wang, X. Xu, and T. Zhang, "Multipath parallel ICCP underwater terrain matching algorithm based on multibeam bathymetric Data," IEEE Access, vol. 6, pp. 48708-48715, 2018.

[13] J. Zhao, S. Wang, and A. Wang, "Study on underwater navigation system based on geomagnetic match technique," 2009 9th International Conference on Electronic Measurement \& Instruments, Beijing, 2009, pp. 3-255-3-259.

[14] B. Wang, Y. Zhu, Z. Deng, M. Fu, "The Gravity Matching Area Selection Criteria for Underwater Gravity-Aided Navigation Application Based on the Comprehensive Characteristic Parameter," IEEE/ASME Transactions on Mechatronics, vol. 21, no. 6, pp. 2935-2943, Dec. 2016.

[15] L. Zhao, N. Gao, B. Huang, et al., "A novel terrain-aided navigation algorithm combined with the TERCOM algorithm and particle filter," IEEE Sensors Journal, vol. 15, no. 2, pp. 1124-1131, Sep. 2014.

[16] X. Ma, H. Liu, D. Xiao, et al., " Key technologies of Geomagnetic Aided Inertial Navigation System// Intelligent Vehicles Symposium. IEEE, 2009.

[17] B. Wang, Y. Lu, Z. Deng, M. Fu, "A Particle Filter-Based Matching Algorithm With Gravity Sample Vector for Underwater Gravity Aided Navigation," IEEE/ASME Transactions on Mechatronics, vol. 21, no. 3, pp. 1399-1408, Jan. 2016.

[18] Y. Han, B. Wang, Z. Deng, M. Fu, “A combined matching algorithm for underwater gravity-Aided navigation," IEEE/ASME Transactions on Mechatronics, vol. 23, no. 1, pp. 233-241, Dec. 2018.

[19] M. Geva-Sagiv, L. Las, Y. Yovel, and N. Ulanovsky, "Spatial cognition in bats and rats: From sensory acquisition to multiscale maps and navigation," Nat. Rev. Neurosci., vol. 16, no. 2, pp. 94-108, Jan. 2015.

[20] W. Wiltschko, R. Wiltschko, "Magnetoreception in birds: two receptors for two different tasks," Journal of Ornithology, vol. 148, pp. 61-76, Oct. 2007.

[21] C. Lohmann, K. Lohmann, "Sea Turtles: Navigation and Orientation," Encyclopedia of Animal Behavior, pp. 101-107, Jul. 2010.

[22] Putman N F . Inherited Magnetic Maps in Salmon and the Role of Geomagnetic Change[J]. Integrative and Comparative Biology(3):3.

[23] L. Boles, K. Lohmann. "True navigation and magnetic maps in spiny lobsters," Nature, vol. 421, no. 6918, pp. 60-63, 2003.

[24] Z. Zhao, T. Hu, W. Cui, J. Huangfu, C. Li, and L. Ran, "Long-distance geomagnetic navigation: Imitations of animal migration based on a new assumption," IEEE Trans. Geosci. Remote Sens., vol. 52, no. 10, pp. 6715-6723, Oct. 2014.

[25] X. K. Qi, D. X. Ye, Y. Z. Sun, C.Z. Li, and R. X. Ran, "Simulations to True Animals' Long-Distance Geomagnetic Navigation," IEEE Transactions on Magnetics, vol. 53, no. 1, pp. 1-8, Jan. 2017.

[26] Y. Zhang, X. Liu, M. Liu, and C. Yang. "Bio-Inspired Approach for Long-Range Underwater Navigation Using Model Predictive Control," IEEE Transactions On Cybernetics, 2019.

[27] H. Li, M. Liu, K.Liu, "Multi-objective evolutionary search algorithm for geomagnetic bio-inspired navigation," Journal of National University of Defense Technoligy, vol. 39, no. 6, pp. 98-102, Dec. 2017.

[28] H. Li, M. Liu, K. Liu, "Bio-inspired geomagnetic navigation method for autonomous underwater vehicle," Journal of Systems Engineering and Electronics, vol. 28, no. 6, pp.1203-1209, Dec 2017.

[29] J. Aubert, J. A. Tarduno, and C. L. Johnson. "Observations and models of the long-term evolution of Earth's magnetic field," Space Sci. Rev., vol. 155, nos. 1-4, pp. 337-370, Aug. 2010.

[30] S. Maus and H. Lühr, "Signature of the quiet-time magnetospheric magnetic field and its electromagnetic induction in the rotating Earth," Geophys. J. Int., vol. 162, no. 3, pp. 755-763, Jul. 2005.

[31] G. A. Glatzmaier and P. H. Roberts, "Rotation and magnetism of Earth's inner core," Science, vol. 274, no. 5294, pp. 1887-1891, Dec. 1996.

[32] J. Bloxham, D. Gubbins, and A. Jackson, "Geomagnetic secular variation," Philos. Trans. R. Soc. London, vol. 329, no. 1606, pp. 415-502, Nov. 1989.

[33] Z. Liu, M. Pan, Y. Tang, et al. "A new method for distortion magnetic 
field compensation of a geomagnetic vector measurement system," Measurement Science and Technology, vol. 27, no. 12, 2016.

[34] A. T. Y. Lui, "Tutorial on geomagnetic storms and substorms," IEEE Trans. Plasma Sci., vol. 28, no. 6, pp. 1854-1866, Dec. 2000.

[35] Q. Wang, J. Zhou, "Geomagnetic Gradient Bionic Navigation Based on Parallel Approach Method," Journal of Northwestern Polytechnical University, vol. 36, no. 4, pp. 611-617, Aug. 2018.

[36] H. Li. "Research on the geomagnetic bio-inspired navigation for AUV based on optimization searching strategy," Ph.D. dissertation, Dept. School of marine science and technoligy., Northwestern Polytechnical Univ. Xian, China, 2018.

[37] Y. Gao, J. Liu. "Parameter study of differential evolution algorithm," Journal of natural science of Heilongjiang university, vol.24, no. 2, pp. 81-85, Feb. 2009.

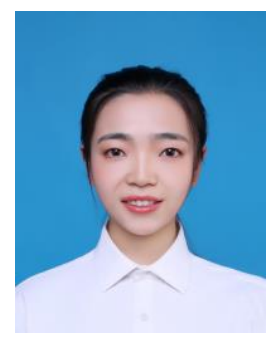

Jiayu Zhang received the M.S. degree in electronic science and technology from the School of Instrument and Electronics, North University of China, Taiyuan, China, in 2019. She is currently pursuing the Ph.D. degree in instrument science and technology with Southeast University, Nanjing, China. Her current research interests include inertial navigation and geomagnetic navigation.

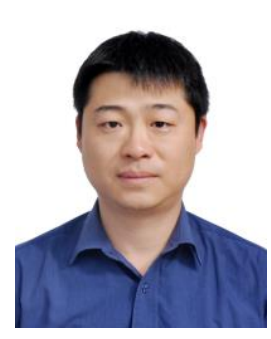

Tao Zhang received Ph.D. degree in navigation, guidance, and control from Southeast University, Nanjing, China, in 2008.

$\mathrm{He}$ is currently a professor with the School of Instrument Science and Engineering, Southeast University, Nanjing, China. His current research interests include inertial navigation, and integration navigation, based on AUV underwater.

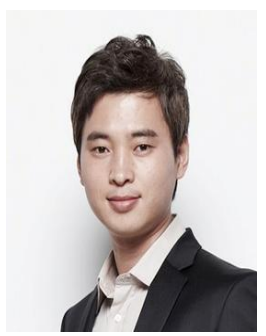

Hyo-Sang Shin received his BSc from Pusan National University in 2004 and gained an MSc on flight dynamics, guidance and control in Aerospace Engineering from KAIST and a PhD on cooperative missile guidance from Cranfield University in 2006 and 2010, respectively. $\mathrm{He}$ is currently a Professor on Guidance, Control and Navigation Systems in Autonomous and Intelligent Systems Group at Cranfield University. His current research interests include multiple target tracking, adaptive and sensor-based control, and distributed control of multiple agent systems.

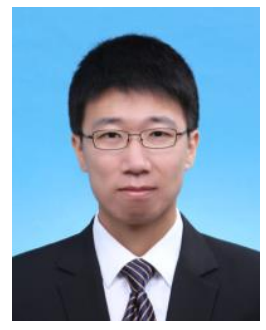

Jian Wang received M.S. degree from Harbin Engineering University, Harbin, China, in 2016. $\mathrm{He}$ is currently pursuing the Ph.D. degree in instrument science and technology with Southeast University, Nanjing, China. His current research interests include SINS initial alignment, SINS/USBL integration navigation, et al.

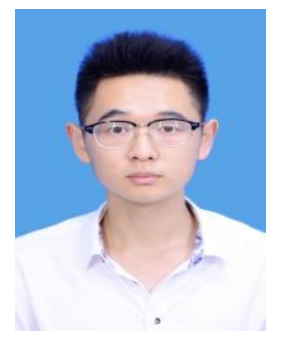

Chen Zhang received the B.E. degree in measurement and control technology and instrumentation from Jiangsu University, Jiangsu, China, in 2019. He is a M.E. candidate in navigation guidance and control from Southeast University, Nanjing, China. His current research interests include geomagnetic navigation and magnetic calibration. 
$2020-10-30$

\section{Geomagnetic gradient-assisted} evolutionary algorithm for long-range underwater navigation

\section{Zhang, Jiayu}

IEEE

Zhang J, Zhang T, Shin HS, et al., (2020) Geomagnetic gradient-assisted evolutionary algorithm for long-range underwater navigation. IEEE Transactions on Instrumentation and Measurement, Volume 70, October 2020, Article number 2503212

https://doi.org/10.1109/TIM.2020.3034966

Downloaded from Cranfield Library Services E-Repository 